\title{
Promoting Community-based Engagement with Pregnant and Postpartum Women with Substance Use Disorder via Human-centered Design
}

\author{
Scott Liu' ${ }^{1}$ Brandon Cockrum², Courtney Moore², Gina Claxton², Lisa Parks², Sarah Wiehe ${ }^{1,2,3,4}$, \\ Joanna Chambers ${ }^{1,2,3}$, Scott Denne ${ }^{1,2,3}$, Debra Litzelman ${ }^{1,2,3,4}$ \\ ${ }^{1}$ Indiana University School of Medicine, ${ }^{2}$ Indiana Clinical and Translational Sciences Institute \\ ${ }^{3}$ Indiana University Health, ${ }^{4}$ Regenstrief Institute
}

\section{Background:}

Pregnant women with substance use disorder (SUD) are a vulnerable population that often contends with stigma, socioeconomic inequity, and mental health issues. The $\underline{\text { Community-based }}$ Addiction Reduction (CARE) Program connects pregnant and postpartum women with SUD in Central Indiana with addiction recovery coaches (ARCs) and attachment theory therapists, who provide linkage to community resources and mental health support. However, recruitment and retention of participants continue to be challenging.

The goals of this research are to identify barriers and facilitators to recruitment/retention and to translate findings into patient engagement materials.

\section{Methods:}

ARCs are women with family or personal experience with SUD, and serve as proxies for patient perspectives. Qualitative data from nine semi-structured interviews conducted with ARCs and their supervisors were clustered thematically to identify barriers and facilitators to recruitment/retention. Rapid prototyping, a methodology within human-centered design (HCD), was then employed to iteratively develop deliverables, incorporating several rounds of critique from ARCs, weekly case conferences, and discussions with clinician-investigators and designers.

\section{Results:}

Facilitators to recruitment/retention included: (1) ability to personally relate to ARCs and (2) ARC advocacy when navigating community and health system resources. Barriers to recruitment/retention included: (1) distrust (associated with participants' histories of adverse childhood events and other trauma); (2) overwhelming number of interactions with various agencies (DCS, clinics, counseling, etc.); (3) fluctuating phone access, (4) intensive enrollment process, and (5) overall program visibility.

The following deliverables were designed: (1) introduction videos of ARCs (2) brief videos of patient advocacy stories told by ARCs, (3) personalized cards paired with existing gift card incentives, and (4) journal for self-reflection and self-motivation.

\section{Potential Impact:}

Qualitative findings underscore the importance of patient-centeredness in engagement with CARE participants, who are often overwhelmed and wary of others. HCD approaches within 
multidisciplinary teams can be leveraged to enhance program uptake and delivery within underserved populations. 